PAEDIATRIC LUNG DISEASE

\title{
Cough frequency in children with stable asthma: correlation with lung function, exhaled nitric oxide, and sputum eosinophil count
}

\author{
A M Li, C Lex, A Zacharasiewicz, E Wong, E Erin, T Hansel, N M Wilson, A Bush
}

Thorax 2003;58:974-978

See end of article for authors' affiliations

Correspondence to:

Dr A Bush, Professor in

Paediatric Respiratory

Medicine, Royal Brompton

Hospital, Sydney Street,

London SW3 6NP, UK;

a.bush@rbh.nthames.

nhs.uk

Revised version received 5 April 2003

Accepted for publication

28 July 2003
Background: A study was undertaken to assess the correlation between cough frequency in asthmatic children with lung function and two non-invasive markers of airway inflammation.

Methods: Thirty two children of median age 12.0 years (interquartile range (IQR) 9.5-13.4) with stable asthma were recruited. They underwent spirometric testing, exhaled nitric oxide (eNO) measurement, sputum induction for differential cell count, and ambulatory cough monitoring over 17 hours and 40 minutes. Coughing episodes were counted both as individual spikes and as clusters.

Results: Complete cough frequency data were available in 29 children $(90 \%)$ and their median forced expiratory volume in 1 second $\left(\mathrm{FEV}_{1}\right)$ and eNO were 88.5\% (IQR 79.5-98) and 23.9 ppb (IQR 11.441.5), respectively. The median number of cough episodes was 14 (IQR 7.0-24.0) which was significantly higher than that of normal children (6.7 (IQR 4.1-10.5), $\mathrm{p}<0.001)$. Sputum induction was successful in $61 \%$ of the subjects; the median induced sputum eosinophil count was $0.05 \%$ (IQR 0-9.0). Cough frequency was found to have a significant positive correlation with eNO (Spearman's $r=0.781, p<0.001$ ) but not with $\mathrm{FEV}_{1}$ or sputum eosinophil count $(r=-0.270, \mathrm{p}=0.157 ; r=0.173, \mathrm{p}=0.508$, respectively). Conclusions: Children with stable asthma have increased cough frequency compared with normal controls and cough frequency was greater during the day than at night. Cough may be a more sensitive marker of airway inflammation than simple spirometry.
C ough in a community context is common and is rarely a manifestation of asthma. Cough is also a very common symptom in children with asthma and rarely can be the only symptom. Subjective cough assessment is unreliable and a major difficulty has been our inability to quantify cough accurately. ${ }^{1-3}$ The use of diary cards has been criticised for the lack of repeatability and reliability. ${ }^{45}$ Previous investigators have used conventional tape recorders to quantify cough objectively, but their bulk and lack of portability preclude their use in daytime cough assessments during normal activity. Furthermore, they rely exclusively on an audio signal. Notwithstanding these difficulties, the assessment of cough frequency is commonly included in asthma severity scales. ${ }^{6}$ It is known that, in some children, cough precedes the onset of an asthma exacerbation. ${ }^{7}$ Furthermore, in adults with cough variant asthma and eosinophilic bronchitis, it has been shown that cough can be the only manifestation of eosinophilic airway inflammation. ${ }^{8}$ However, the relation between frequency of cough in children with asthma and airway inflammation is unknown.

We hypothesised that cough frequency is more sensitive to airway inflammation than is spirometry. In other words, a child may have an inflamed airway with an abnormal cough frequency and yet have normal lung function. The aim of this study was twofold: (1) to measure cough frequency in stable asthmatic children and (2) to assess the correlation between cough frequency and the following parameters: (a) spirometry, (b) exhaled nitric oxide (eNO), and (c) eosinophil count in induced sputum. An ambulatory cough monitoring device (LR 100) was used to measure cough frequency. This system has been validated in children and found to be highly acceptable, with no adverse effects having been reported during recording. ${ }^{10}$

\section{METHODS}

\section{Subjects}

All children with stable asthma were recruited from the Paediatric Asthma Clinic at the Royal Brompton Hospital. The children were aged 7-18 years and all were able to cooperate with the tests. The diagnosis of asthma was made on standard grounds. ${ }^{11}$ Stable asthma was defined as no asthmatic exacerbation for the preceding 4 weeks necessitating oral prednisolone or an increased use of inhaled corticosteroid, the use of rescue treatment no more than three times a week, and with no clinical indication for change in treatment medication. Children who had wheeze due to concomitant non-asthmatic chronic airway diseases such as cystic fibrosis, respiratory conditions apart from asthma that could have caused cough, or patients with asthma who had suffered an exacerbation within a month of the study period were excluded.

Approval for the study was obtained from the ethics committee of the Royal Brompton Hospital and Harefield NHS Trust. The children's parents or guardians gave informed consent and age appropriate assent was obtained from the child.

\section{Exhaled NO}

Each child was examined and height (Harpenden stadiometer) and weight (minimal underwear, digital Seca scale) were recorded. Exhaled NO (eNO) was measured before baseline spirometric tests using a chemiluminescence analyser (NIOX, Aerocrine, Stockholm, Sweden) according to the American Thoracic Society guidelines. ${ }^{12}$ The analyser is sensitive to NO from 0 to $200 \mathrm{ppb}$ (response time $<1.5 \mathrm{~s}$ ). Children breathed room air and kept the flow during expiration within a constant range by use of visual guides. The expiratory pressure was $5-15 \mathrm{~cm} \mathrm{H}_{2} \mathrm{O}$ and the flow rate 
$50 \mathrm{ml} / \mathrm{s}$. Measurements were made when NO levels achieved a stable plateau phase for at least 2 seconds. The mean value out of three technically acceptable manoeuvres was recorded.

\section{Pulmonary function}

Spirometric tests were performed at baseline and 1 min after each inhalation period during sputum induction. A portable spirometer (Compact Vitalograph, Vitalograph Ltd, Buckingham, UK) was used according to the American Thoracic Society guidelines. ${ }^{13}$ Subjects were allowed to continue their own anti-asthma medication, but were asked to withhold short and long acting bronchodilators for 4 and 12 hours, respectively, if applicable. Three technically acceptable manoeuvres were performed each time and the highest value of forced expiratory volume in 1 second $\left(\mathrm{FEV}_{1}\right)$ was recorded. Values were expressed as a percentage of predicted normal values. The subjects were allowed to proceed to sputum induction if the baseline $\mathrm{FEV}_{1}$ was greater than $65 \%$ predicted.

\section{Sputum induction and processing}

Sputum induction was performed using an inhalation of $4.5 \%$ hypertonic saline (HS) through a mouthpiece and a large two-way valve (Hans Rudolph Inc, Kansas City, USA) connected to a DeVilbiss ultrasonic nebuliser (Somerset) set at the maximum output $(6 \mathrm{l} / \mathrm{min})$. The procedure was explained to the child who rinsed his or her mouth with water to clear debris and squamous epithelial cells. A noseclip was worn and baseline $\mathrm{FEV}_{1}$ and forced vital capacity (FVC) were measured. The child then inhaled $4.5 \%$ HS for a period of $30 \mathrm{~s}$. Lung function was repeated $1 \mathrm{~min}$ later. If no sputum was obtained and lung function was greater than $80 \%$ of the baseline value, the test was continued. The child then continued inhalation of HS for periods of $1 \mathrm{~min}, 2 \mathrm{~min}$, and then three periods of 4 min each. The child was encouraged to cough up any sputum after each dose of HS and the sputum was collected into a $50 \mathrm{ml}$ polypropylene tube, kept at $4^{\circ} \mathrm{C}$, and processed within 2 hours. A record of any side effects experienced by the child undertaking the test was made at the end of each elapsed time period. The study concluded either when the child developed troublesome symptoms, when lung function had dropped below $80 \%$ of the baseline value, or if the child could not be persuaded to complete the whole inhalation procedure. If the child had a greater than $20 \%$ fall in $\mathrm{FEV}_{1}, 500 \mu \mathrm{g}$ salbutamol was administered using a metered dose inhaler and spacer and recovery monitored. The subject was then allowed to proceed to the next stage of assessment once the $\mathrm{FEV}_{1}$ had returned to baseline.

For sputum processing the sputum was poured onto a Petri dish and all solid material, usually gelatinous and mucoid, was selected from the salivary fluid with blunt ended forceps. The weight of the selected sputum was measured and $0.1 \%$ dithiothreitol (DTT) (Sigma Chemicals, Poole, UK) was added to the sample in the ratio of $4 \mathrm{ml}$ DDT to $1 \mathrm{~g}$ sputum. The mixture was vortexed and repeatedly aspirated at room temperature until the sputum was homogenised. The sample was then filtered using a $48 \mu \mathrm{m}$ pore nylon mesh filter and phosphate buffered saline (PBS) was added to the filtrate in a ratio of $4 \mathrm{ml} \mathrm{PBS}$ to $\mathrm{lg}$ original sputum weight. The preparation was vortexed briefly and centrifuged at $400 \mathrm{~g}$ for $10 \mathrm{~min}$ at $4^{\circ} \mathrm{C}$. The supernatant was aliquoted into labelled cryotubes which were stored at $-70^{\circ} \mathrm{C}$ for future analysis. The cell pellets from the sputum centrifugation were resuspended for total cell counts recorded with a haemocytometer using trypan blue stain. Slides were prepared using a cytospin instrument (Shandon, Runcorn, UK) and stained with Diff-Quik stain (Dade Behring, UK) for differential cell counts. All slides were coded before counting and the personnel involved were unaware of the eNO and cough frequency results of the subjects. At least 400 inflammatory cells were counted in each sample and an adequate sample was defined as having less than $50 \%$ squamous epithelial cells in a cytospin preparation. The eosinophil count was expressed as a percentage of the total cell count.

\section{Cough monitoring}

The LR 100 cough recorder used in this study is a modification of our ambulatory cough monitoring device which has been validated in adults and children. ${ }^{9}{ }^{10}$ The recording time is 17 hours and 40 minutes and the machine stops automatically when the recording period has elapsed. LR 100 is a multiparametric recording device worn in a waist bag connected to the chest by three electromyographic (EMG) leads and a microphone. Two signals are recorded, a surface EMG and an audio signal, and cough is defined as before by a combination of rapid phasic bursts in both signals. The EMG signal processing is such that voluntary muscle movement is filtered out and the rapidly recruited abdominal wall muscles involved in coughing are recorded. The audio signal of the cough is recorded simultaneously, thus giving two signals from which to verify that the child has coughed. The information obtained is played back and displayed simultaneously visually on a personal computer. All data collected were inspected and analysed both automatically and visually. The modification here was the use of the data analysis by preset algorithms. The previous monitor (RBC-7) could only be hand analysed, a laborious progress. The modification shortened analysis time by several hours by automatically discarding any period of the recordings which clearly contained no cough. ${ }^{10}$ Visual inspection confirmed that all cough epochs identified by the algorithms were in fact genuine. Coughing events were counted both as individual spikes and as clusters or bouts. We arbitrarily defined each cluster (a cough epoch) as a close succession of coughs ( $<2$ seconds between individual coughs) recorded by each trigger of the recorder. The cough data were expressed as the total number of cough episodes (individual spike+cough cluster) per recording time. The subjects had the monitor attached at $\mathbf{1 8 . 0 0}$ hours on the study day and were asked to return at noon the next day for the removal of the monitor.

\section{Statistical analysis}

The descriptive statistics were expressed as median and interquartile range. Spearman's correlation was used to assess the relation between frequency of cough and lung function and airway inflammation. The Mann-Whitney test was used to compare non-parametric data. All tests were performed using SPSS for Windows (Release 11.0; SPSS Inc, Chicago, Illinois, USA), and the level of significance was set at $5 \%$ for all comparisons.

\section{RESULTS}

Thirty two children of median age 12.0 years (range 9.5-13.4) with stable asthma were recruited. All except one patient was using inhaled corticosteroid and the median beclomethasone equivalent dosage was $1144 \mu \mathrm{g}$ (range 0-4000 $\mu \mathrm{g}$, assuming budesonide has equal potency and fluticasone is twice as potent as beclomethasone). Twenty six patients were using a long acting $\beta$ agonist and eight were taking regular leukotriene receptor antagonist. Five patients were also prescribed systemic corticosteroids and other medications used were theophylline $(n=4)$, nedocromil sodium $(n=1)$, and sodium cromoglycate $(n=1)$. Their median FEV $_{1}$ was $88.5 \%$ predicted (range $79.5-97.5$ ). The median eNO was $23.9 \mathrm{ppb}$ (range 11.4-41.4). Mean (2 SD) eNO taken from a group of normal healthy children with a mean age of 10.7 
Table 1 Demographic characteristics of study children

\begin{tabular}{ll}
\hline & Median (IQR) \\
\hline Age (years) & $12.0(9.5-13.4)$ \\
Age asthma diagnosed (years) & $1.50(0.9-3.8)$ \\
Weight (kg) & $41.0(34.0-49.3)$ \\
Height (cm) & $145.9(135.8-155.7)$ \\
eNO (ppb) & $23.9(11.4-41.5)$ \\
FEV $1 \%$ predicted) & $88.5(79.5-97.5)$ \\
FVC (\% predicted) & $107.0(94.25-118.75)$ \\
PEFR (\% predicted) & $85.0(79.25-100.75)$ \\
\hline eNO = exhaled nitric oxide; FEV & = forced expiratory volume in 1 second; \\
FVC = forced vital capacity; PEFR = peak expiratory flow rate; \\
IQR = interquartile range.
\end{tabular}

years was 15.6 (9.2) ppb. ${ }^{14}$ The baseline characteristics of the children are summarised in table 1.

\section{Cough frequency assessment}

Complete cough frequency data were available in 29 children (90\%) and no adverse events or sleep disturbance were reported. In two subjects the EMG leads stopped functioning during recording and in one subject the data from the cough monitor were unretrievable. A previous study using a similar monitoring device recorded cough frequency on 44 normal children aged 8-12 years and found a median of 6.7 episodes per 18 hours (IQR 4.1-10.5). ${ }^{9}$ In our study the median number of cough episodes was 14 (IQR 7-24) which was statistically greater than that of normal controls $(\mathrm{p}<0.001)$. There was no correlation between age and cough frequency (Spearman's correlation coefficient $=-0.295$ (confidence interval -0.597 to 0.080$), p=0.12$ ). The distribution of cough episodes over the whole recording period is shown in fig 1 .

We also attempted to compare night and daytime cough frequency. We were not able to measure sleep stage, so we arbitrarily defined night cough as cough occurring between midnight and 07.00 hours, and daytime cough as cough occurring at any other time. We calculated the hourly cough frequency for these two periods. Median daytime coughs/ hour were significantly greater than night time coughs (39.5/ hour (IQR 25.5-57.25) versus 11.5/hour (IQR 6.25-17.5), $\mathrm{p}<0.001)$. We further analysed the data using the time of 21.00 hours and 22.00 hours as cut-offs for night and day coughs, but this did not alter our conclusions.

\section{Sputum induction}

Sputum induction was tolerated by all subjects and no adverse effects were reported. Twenty subjects (61\%) were

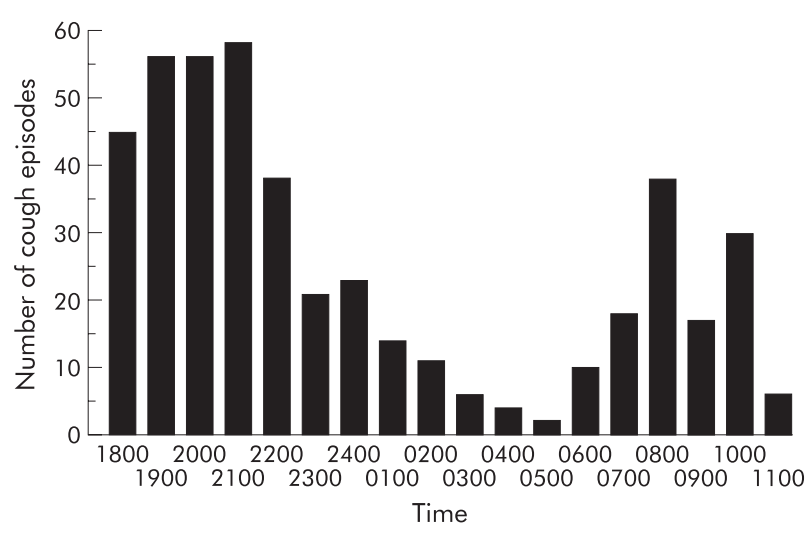

Figure 1 Cough frequency distribution. able to produce an adequate sputum sample. The median weight of the sputum sample was $0.94 \mathrm{~g}$ (IQR 0.76-1.34) and the median eosinophil count was $0.05 \%$ (range $0-9.0 \%$ ). Median sputum eosinophil count obtained from a cohort of healthy children was found to be $1 \%$ (IQR $0.3-1.8){ }^{15}$

\section{Correlation with spirometry and airway inflammation markers}

Using Spearman's correlation, cough frequency was found to have a significant positive correlation with eNO (fig 2A) but not with $\mathrm{FEV}_{1}$ (fig 2B) or sputum eosinophil count (fig 2C). There was only a very weak trend for a positive correlation between eNO and sputum eosinophil count (fig 2D). The results are summarised in table 2.

\section{DISCUSSION}

A positive correlation was found between cough frequency and the degree of airway inflammation as assessed by exhaled nitric oxide (eNO), but no correlation was found with lung function in a group of children with chronic asthma. Despite being recruited at a time of apparent stability, they had a higher cough frequency than normal controls. ${ }^{9}$ We cannot comment on whether this increased cough frequency would have responded to an increase in asthma medications, but the point of the study is that, for whatever reasons, cough was increased in children with asthma who were thought clinically to be stable with well controlled disease. An unexpected finding in our study was that cough frequency was greater during the day than at night. Since the children were otherwise asymptomatic, we could not compare cough with other symptoms such as wheeze.

Evidence of airway inflammation in asthmatic patients with cough has previously been reported. Chang et al ${ }^{16}$ related asthma severity to cough and inflammatory markers. Twenty one children (age range 5.5-16 years) were recruited and 11 were followed during days $1,3,7$, and 28 of a mild asthma exacerbation. The authors found that mild asthma exacerbations were characterised by an increase in cough and eosinophilic but not neutrophilic inflammation with nonsignificant changes in lung function. They concluded that, in children with asthma, increased cough mirrors the onset of an exacerbation and increased eosinophilic inflammation. This is in agreement with another study which reported that, in patients with asthma and chronic cough, the airway inflammation was more marked than in patients with chronic cough alone. ${ }^{17}$ eNO as a marker of airway inflammation was found to be significantly associated with reported cough in the previous 12 months. ${ }^{18}$ Unfortunately, none of these studies monitored cough objectively. To our knowledge, this is the first study directly comparing markers of airway inflammation with objective cough data. Persistent airway inflammation in stable asthmatics may explain our finding of increased total cough. A recent study showed that persistent airway inflammation was still evident in asthmatics who had been asymptomatic with normal lung function. ${ }^{19}$ The actual mechanism whereby airway inflammation may cause cough in patients with asthma is still uncertain and this study is not helpful in this regard. We had too few patients using long acting $\beta_{2}$ agonists to make any comment on the effects of these medications on cough. It may be that the release of inflammatory mediators such as bradykinin and prostaglandins enhances the cough reflex. ${ }^{20}{ }^{21}$ It would also appear that subclinical inflammation may be manifest by increased cough before any change in lung function.

The relationship between eNO and sputum eosinophils was poor. The best correlations have been seen in steroid naïve asthmatics, ${ }^{22}$ and all but one of our patients was using inhaled corticosteroids, so this is not surprising. We cannot 

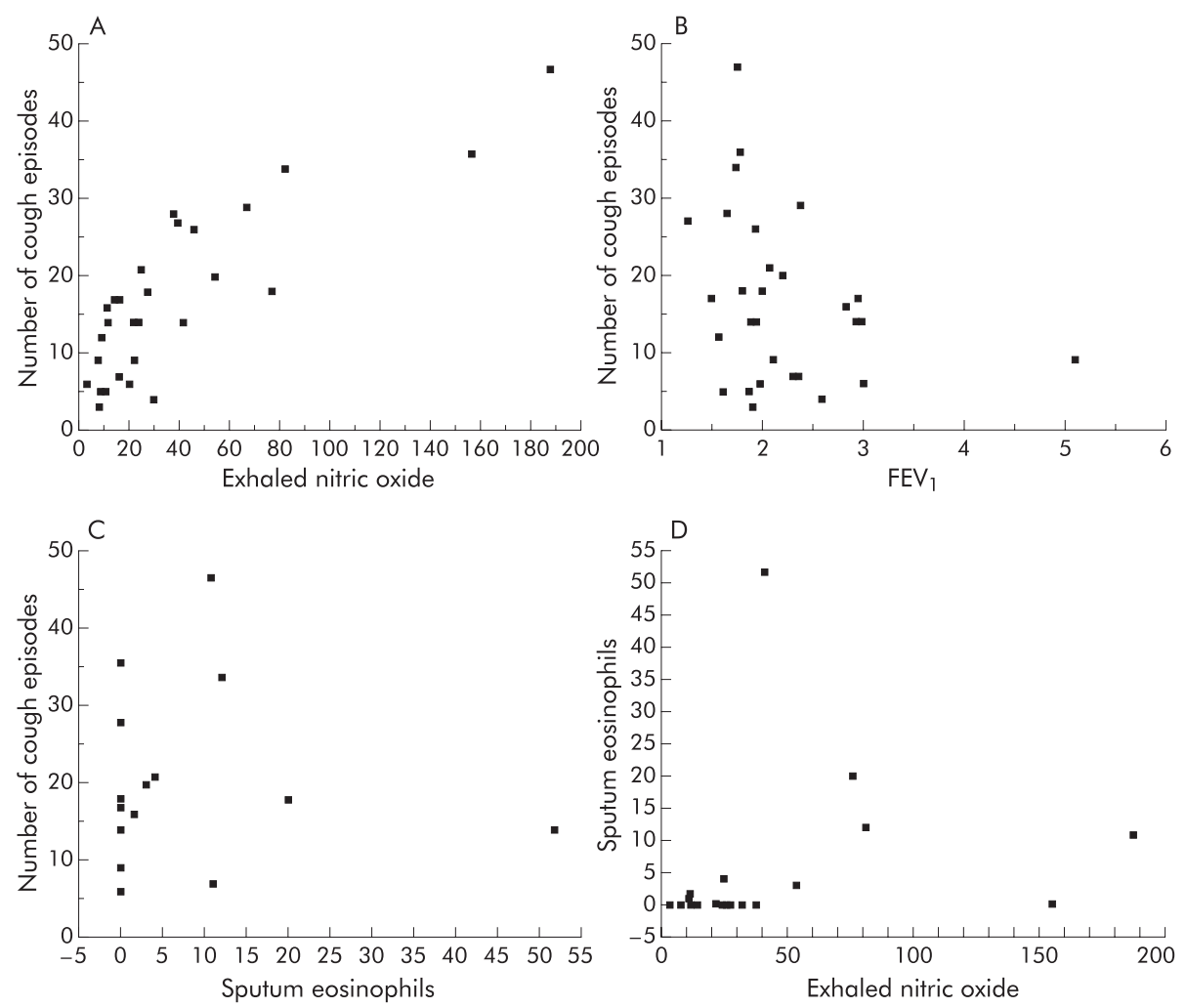

Figure 2 (A) Correlation between cough frequency and eNO; (B) correlation between cough frequency and forced expiratory volume in 1 second $\left(\mathrm{FEV}_{1}\right)$; $(\mathrm{C})$ correlation between cough frequency and sputum eosinophil count (\%); (D) correlation between sputum eosinophil count and eNO.

exclude the possibility that we would have seen a closer relationship between cough and sputum eosinophils in children with milder asthma. This study was not designed to explore the relationship between eNO and cough in normal subjects. An increase in eNO in normal subjects may be due to viral infection or environmental pollution which may also cause cough. ${ }^{23}$ In this group there may be a different relationship between cough and eNO. We focused on asthmatics during clinical stability, and thus tried to avoid these possible confounding effects. In subjects with asthma the severity of cough has been shown to be unrelated to measures of airway calibre. ${ }^{24} \mathrm{Hsu}$ and colleagues showed that, in adults with non-acute asthma, the frequency of cough had no relationship with asthma severity measured by $\mathrm{FEV}_{\mathrm{I}}$ and diurnal peak flow variation. ${ }^{25}$ Indeed, in our study we were not able to show any relationship between cough frequency and spirometry. We did not measure bronchial hyperreactivity or peak flow variability with time and this is a weakness of the study. We were constrained by the number of visits and procedures we could expect of the children. We cannot therefore exclude a relationship between measures of airway lability and cough frequency.

Cough is often part of the symptom complex in children with asthma but data on the cough frequency in these subjects are limited. Tracheal sounds were continuously recorded in the homes of 60 children with and 30 without asthma. The results showed that children with asthma coughed significantly more often than control subjects during exacerbations, but not during remission. ${ }^{26}$ This is in contrast to our study in which we found that children with stable asthma had an increased cough frequency compared with data previously obtained from normal school children. ${ }^{9}$ Furthermore, we have found that cough frequency is greater during the day than at night, despite conventional teaching

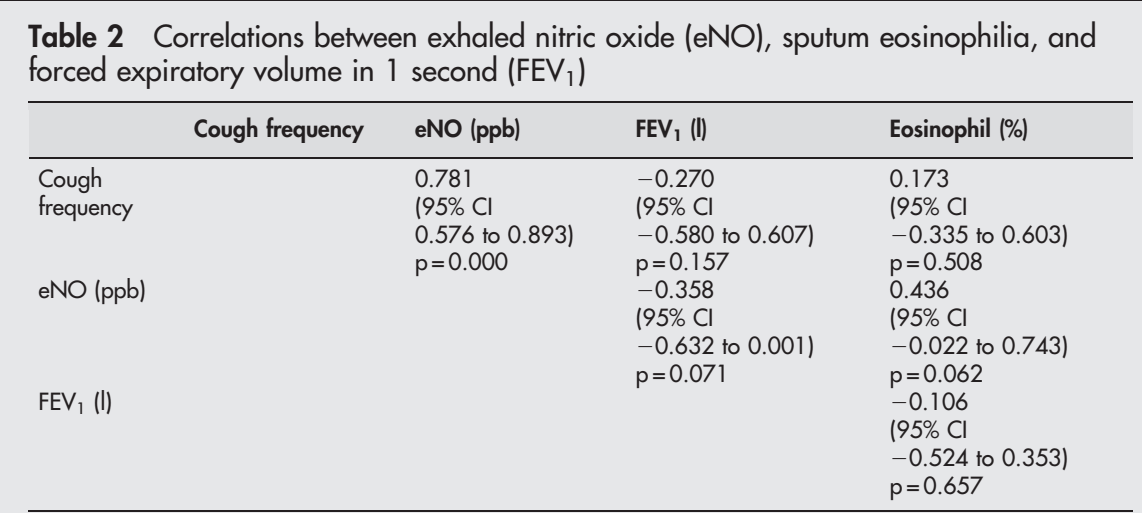

Results are expressed as Spearman's correlations with $95 \%$ confidence intervals and $p$ values. 
on the importance of night cough in asthma. This underscores the need to monitor cough both by day and night if therapeutic interventions directed at alleviating cough are being attempted in asthmatics. The actual timing of cough might reflect differing mechanisms that are dependent on the sites and/or pathology of the underlying respiratory disorder.

There are a number of weaknesses in our study. Firstly, we included a relatively small number of patients. We would have liked to include patients with milder asthma and especially those who are steroid naïve. This would have allowed a better opportunity to study the cough mechanism in relation to different treatment regimens. Our findings may have been different if a larger population of asthmatics with a wide range of severity was included. We also cannot exclude the possibility that a larger sample size including patients with more marked sputum eosinophilia might have resulted in a significant association between the frequency of cough and sputum eosinophil counts. Ideally, we would have liked to use bronchoscopy, bronchoalveolar lavage, and airway biopsy to measure inflammation directly, but this would not be ethical in children. The use of eNO as an airway inflammatory marker has been suggested to be reliable and its correlation with the percentage eosinophils in bronchoalveolar lavage fluid has recently been shown in a group of children with asthma of varying severity. ${ }^{27}$ Because of the limitation of the current version of the cough monitor, we could only monitor for a period of 17 hours and 40 minutes; 24 hour monitoring would provide important information for diurnal comparison of symptoms. We also could not assess sleep stage or even whether the child was asleep, so we could not distinguish night cough from coughing when asleep. The main strength of the study is the use of an objective cough monitor relying on two signals to confirm coughing by the child. The accuracy of tracheal sound recording as the only cough signal is questionable, relying on just an audio signal. Sound quality may be affected by the sleep position of the patient and the actual recordings may be contaminated by sounds made by people nearby. The device has been validated in adults and children ${ }^{9}{ }^{10}$ and found to be user friendly and free from adverse side effects. Using this monitor, the relationship between cough and other asthma symptoms such as sleeplessness, exercise tolerance, and $\beta$ agonist consumption can be further explored.

In conclusion, the frequency of cough in stable asthmatics was increased compared with normal subjects; it was particularly marked during daylight hours and was positively correlated with eNO but not with spirometry. Cough may thus be a more sensitive clinical marker of airway inflammation than simple spirometry. However, longitudinal studies are required to determine whether increasing asthma treatment is a useful strategy to treat increased cough in the context of known asthma. If increased cough frequency is confirmed to be a useful marker of airway inflammation in longitudinal studies, the challenge will be to find cheap and clinically applicable ways of measuring cough objectively as part of the routine assessment of the asthmatic child.

\section{Authors' affiliations}

A M Li, C Lex, A Zacharasiewicz, N M Wilson, A Bush, Department of Paediatric Respiratory Medicine, Royal Brompton Hospital, London SW3 6NP, UK
A M Li, Department of Paediatrics, Chinese University of Hong Kong, Prince of Wales Hospital, Shatin, Hong Kong

E Wong, Centre for Clinical Trials and Epidemiological Research, Chinese University of Hong Kong, Prince of Wales Hospital, Shatin, Hong Kong

E Erin, T Hansel, Clinical Studies Unit, Royal Brompton Hospital, London SW3 6NP, UK

\section{REFERENCES}

1 Archer LNJ, Simpson H. Night cough counts and diary card scores in asthma. Arch Dis Child 1985;60:473-4.

2 Falconer A, Oldman C, Helms P. Poor agreement between reported and recorded nocturnal cough in asthma. Pediatr Pulmonol 1993;15:209-11.

3 Brunekreef B, Groot B, Rijcken B, et al. Reproducibility of childhood respiratory symptom questions. Eur Respir J 1992;5:930-5.

4 Juniper EF, Guyatt GH, Dolovich J. Assessment of quality of life in adolescents with allergic rhinoconjunctivitis: development and testing of a questionnaire for clinical trials. J Allergy Clin Immunol 1994;93:413-23.

5 Thompson AH, Pratt C, Simpson H. Nocturnal cough in asthma. Arch Dis Child 1987;62:1001-4

6 Rosier MJ, Bishop J, Nolan T, et al. Measurement of functional severity of asthma in children. Am J Respir Crit Care Med 1994;149:1434-41.

7 Chang AB, Phelan PD, Robertson CF. Cough receptor sensitivity in children with acute and non-acute asthma. Thorax 1997:52:770-4.

8 Gibson PG, Hargreave FE, Girgis-Gabardo A, et al. Chronic cough with eosinophilic bronchitis: examination for variable airflow obstruction and response to corticosteroid. Clin Exp Allergy 1995;25:127-32.

9 Munyard P, Bush A. How much coughing is normal. Arch Dis Child 1996:74:531-4.

10 Hamutcu R, Francis J, Karakoc F, et al. Objective monitoring of cough in children with cystic fibrosis. Pediatr Pulmonol 2002;34:331-5.

11 Warner JO, Neijens HJ, Landau LI, et al. Asthma: a follow-up statement from an international paediatric consensus group. Arch Dis Child 1992;67:240-8.

12 American Thoracic Society. Recommendations for standardised procedures for the on-line and off-line measurement of exhaled lower respiratory nitric oxide and nasal nitric oxide in adults and children: 1999. Am J Respir Crit Care Med 1999;160:2104-17.

13 American Thoracic Society. Standardization of spirometry: 1994 update. Am J Respir Crit Care Med 1995;152:1 107-36

14 Kharitonov SA, Gonio F, Kelly C, et al. Reproducibility of exhaled nitric oxide measurements in healthy and asthmatic adults and children. Eur Respir J 2003;21:433-8.

15 Gibson PG, Simpson JL, Hankin R, et al. Relationship between induced sputum eosinophils and the clinical pattern of childhood asthma. Thorax 2003;58:116-21.

16 Chang AB, Harrhy VA, Simpson J, et al. Cough, airway inflammation and mild asthma exacerbation. Arch Dis Child 2002;86:270-5.

17 Rytila P, Metso T, Petays T, et al. Eosinophilic airway inflammation as an underlying mechanism of undiagnosed prolonged cough in primary healthcare patients. Respir Med 2002;96:52-8.

18 Steerenberg PA, Janssen NAH, Meer G, et al. Relationship between exhaled $\mathrm{NO}$, respiratory symptoms, lung function, bronchial hyperresponsiveness, and blood eosinophilia in school children. Thorax 2003;58:242-5.

19 van den Toorn LM, Overbeek SE, de Jongste JC, et al. Airway inflammation is present during clinical remission of atopic asthma. Am J Respir Crit Care Med 2001; 164:2107-13.

20 Nichol GM, Nix A, Barnes PJ, et al. Enhancement of capsaicin-induced cough by inhaled prostaglandin $\mathrm{F}_{2 \mathrm{a}}$ : modulation by beta-adrenergic agonist and anticholinergic agent. Thorax 1990;45:694-8.

21 Fox AJ, Lalloo UG, Bernareggi M, et al. Bradykinin and captopril-induced cough in guinea-pigs. Nature Med 1996;2:814-7.

22 Berlyne GS, Parameswaran K, Kamada D, et al. A comparison of exhaled nitric oxide and induced sputum as markers of airway inflammation. J Allergy Clin Immunol 2000; 106:638-44.

23 Franklin P, Dingle P, Stick S. Raised exhaled nitric oxide in healthy children is associated with domestic formaldehyde levels. Am J Respir Crit Care Med 2000;161:1757-9.

24 Hoskyns EW, Heaton DM, Beardsmore CS, et al. Asthma severity at night during recovery from an acute asthmatic attack. Arch Dis Child $1991 ; 66: 1204-8$.

25 Hsu JY, Stone RA, Logan-Sinclair RB, et al. Coughing frequency in patients with persistent cough assessment using a 24 -hour ambulatory recorder. Eur Respir J 1994;7:1246-53.

26 Rietveld S, Rijssenbeek-Nouwens LHM. Diagnostics of spontaneous cough in childhood asthma. Chest 1998;113:50-4.

27 Warke TJ, Fitch PS, Brown V, et al. Exhaled nitric oxide correlates with airway eosinophils in childhood asthma. Thorax 2002;57:383-7. 